\title{
Abatement figures confirmed
}

NHS England and the Chief Dental Officer England have published details setting out key NHS contractual issues going back to the period of lockdown and those applying in the current face-to-face period since 8 June.

The letter, published after extensive discussions between the BDA and NHS England, says that the abatement to be applied to most contracts from 1 April up until 8 June will be $16.75 \%$. This figure is based on an approximate assessment of variable costs that will not have been incurred when practices were closed to faceto-face care (such as laboratory costs and materials).

For practices that have operated as urgent care centres during that period, there will be no abatement for the time they have been providing urgent care. That different approach recognises the extra costs for UDCs, on set-up, protective equipment and potentially staffing.

For the period from 8 June onwards, when practices have begun to provide face-to-face care again, $100 \%$ of contract value will be paid, with no abatement. This recognises the additional costs of providing care during the current period, again for example in terms of PPE. Practices are expected now to be making all possible efforts to be delivering as comprehensive a service as possible to patients.

\section{'NHS England and the Chief Dental Officer England have published details setting out key NHS contractual issues going back to the period of lockdown and those applying in the current face- to-face period since 8 June.'}

Practices will not be measured on the delivery of Units of Dental Activity during this period. However specific assurances will be requested that individual practices are open for face-to-face care, are adhering to contractual hours with reasonable staffing levels for NHS services in place and are performing the 'highest possible levels of activity', with no undue priority being given to private activity over NHS activity.

Practices will need to deliver at least 20\% of usual volumes of patient care activity to receive their full contract value. Importantly, we have agreed with NHS England that the definition of patient care activity includes all clinical contact that dentists or dental care professionals have with patients either face to face, by telephone or video consultation counted by the BSA via completed courses of treatment or via the COVID-19 triage portal.

The period to be assessed will be all clinical contact that dentists or dental care professionals have with patients either face to face, by telephone or video consultation from 20 July to 16 September 2020. This will be compared to the number of courses of treatment only, provided by the contractor in the equivalent period in 2019. Remote advice and care is included in the calculation for 2020 but not for the equivalent period in 2019 . If practices are unable to meet the $20 \%$ threshold, then they will be required to revert to payment based on UDAs.

\section{Wales: Patient numbers to fall off a cliff as COVID-19 hits service already in crisis}

BDA Wales has warned the Welsh

Parliament's Health, Social Care and Sport Committee that without fundamental ongoing reform, dental services in Wales could be fatally compromised by the pandemic.

In oral evidence this morning Welsh dental leaders underlined the risk to practices getting ready to resume routine care, but at a fraction of their former capacity while facing significantly higher costs. Personal Protective Equipment (PPE) remains in short supply, and costs to deliver a single course of treatment have increased by up to $6000 \%$.

Dentists stressed the impact of these overheads on private dentistry, that has been left out on a limb by the Welsh Government in terms of business support, warning their patients have literally nowhere to go if these practices fail. The majority of practices are 'mixed', combining private and NHS work, and therefore will struggle despite support for the NHS side of their business.

Practices in England have resumed routine care on 8 June, but with the overwhelming majority running at less than a quarter of their pre-pandemic capacity. Practices in Wales had remained open during the pandemic and were able to provide a limited menu of emergency treatment.

Whilst the suspension of the discredited 'Units of Dental Activity' model has been welcomed, the BDA is calling for further work to replace the failed system that had demoralised the workforce and fuelled access problems in communities across Wales.

Last year the BDA found only one in 6 practices (55 out of 355 or $15.5 \%$ ) across Wales were able to offer appointments to new adult NHS patients, with only $27 \%$ (96) practices taking on new child NHS patients.

The Committee has previously recommended jettisoning the current contract and development of a new prevention-focused model for care.

Tom Bysouth, Chair of the British Dental Association Welsh General Dental Practice Committee said: 'Dentistry in Wales was in crisis before this pandemic. Now COVID-19 has demolished the flawed foundations the service was built on.

'For over a decade we have worked with a failed NHS contract that valued only activity, activity that is now impossible to deliver.

'Hundreds of thousands of Welsh citizens have struggled to secure access to dentistry. Now with such limited capacity for the foreseeable future, patient numbers across all aspects of dentistry are set to fall off a cliff.

'Practices cannot survive the combination of higher costs and fewer patients without help. Private dentistry has been left to fend for itself, while many mixed and NHS providers are now operating at a loss. This is not a sustainable situation.

'Ministers have pledged a recovery year for dentistry. It's a positive move, but now is the time to embrace much-needed reform, so we can keep delivering better oral health for Wales.' 